\title{
New Aspects of a Lid-Removal Mechanism in the Onset of an Eruption Sequence that Produced a Large Solar Energetic Particle (SEP) Event
}

Alphonse C. Sterling (NASAMSFC); Ronald L. Moore \& David A. Falconer (MSFC, UAH); and J avon M. Knox (Norfolk State U) 


\section{An Active Region Ejective Eruption}

Active region eruption of 2012 J anuary 23.

Ejective eruption.

GOES class M8.7 flare.

CME has " complexities." Very fast: 2100 km/s.

Results in strong Solar Energetic Particle (SEP) event. ( $1 \mathrm{MeV}$ proton flux of $>10^{3}$ pfu for 43 hrs.)

Only $\sim 1 \%--2 \%$ of all CMEs generate SEPs, so this event is "special."

SEPs not the focus here. See J oshi et al. (2013). Also see Liu et al. (2013) for other interplanetary aspects.

("Lid removal" discussion to follow in a bit.) 


\section{Our Focus: Overview of the eruption onset: Eruption dynamics and magnetic topology}

Al A: Adequate time cadence (145 s) and high spatial resolution (0".6 pixels).

SDO/HMI line-of-sight magnetograms.

On-disk from SDO; limb event from STEREO A.

SDO/AIA, various filters $(304,171,193,211,131,335,94$ Ang).

Two distinct eruptions ("two flux ropes," Li \& Zhang 2013, Cheng et al. 2013); Eruption 1 and Eruption 2.

Eruption 2 includes eruption of a filament.
A. Sterling, Dec 2014
L5, Boulder 
SDO AlA_4 94 23-Jan-2012 00:26:38.120 UT

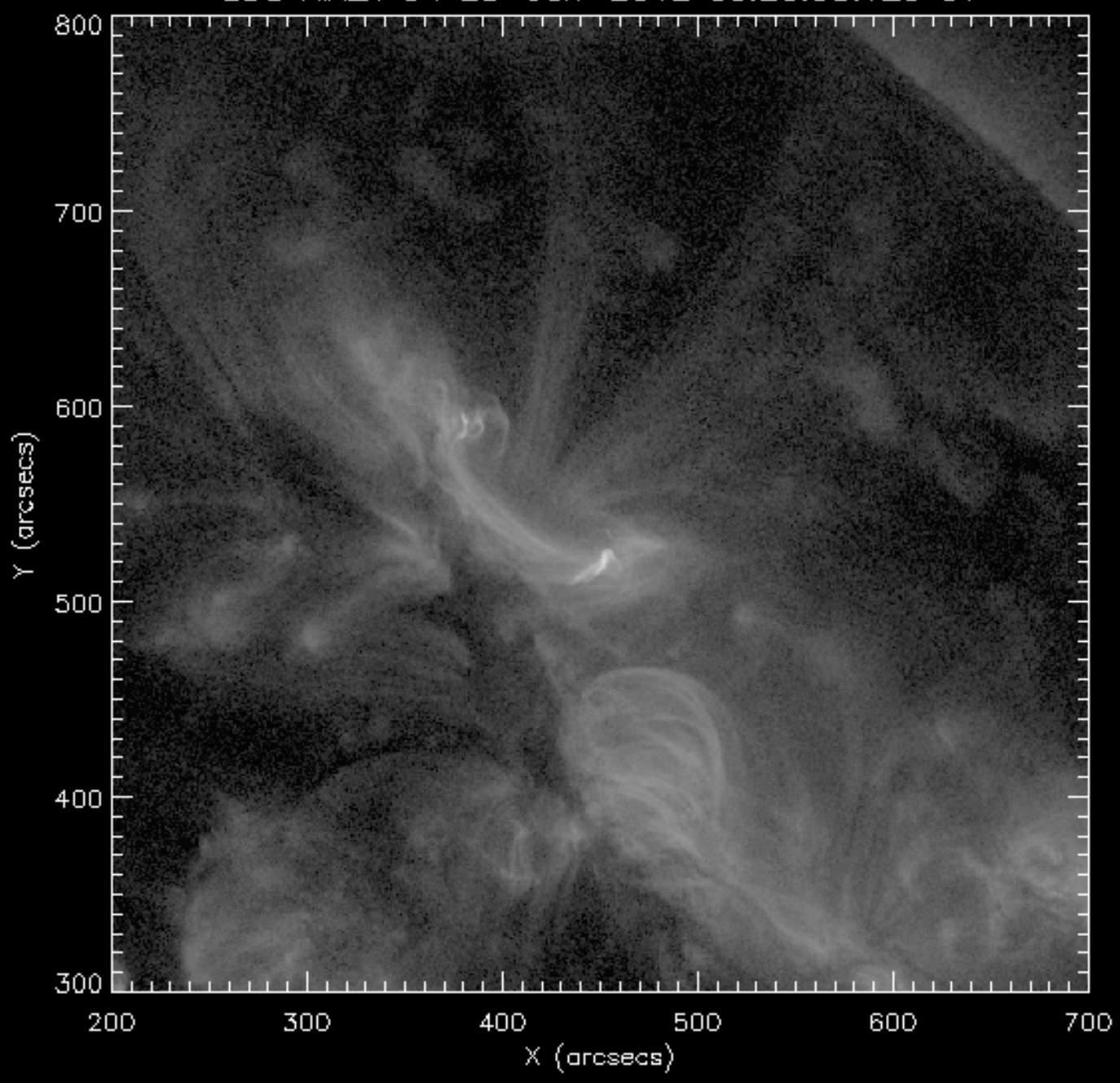

A. Sterling, Dec 2014

L5, Boulder 
SDO AIA 2 193 23-Jan-2012 00:27:07.840 UT

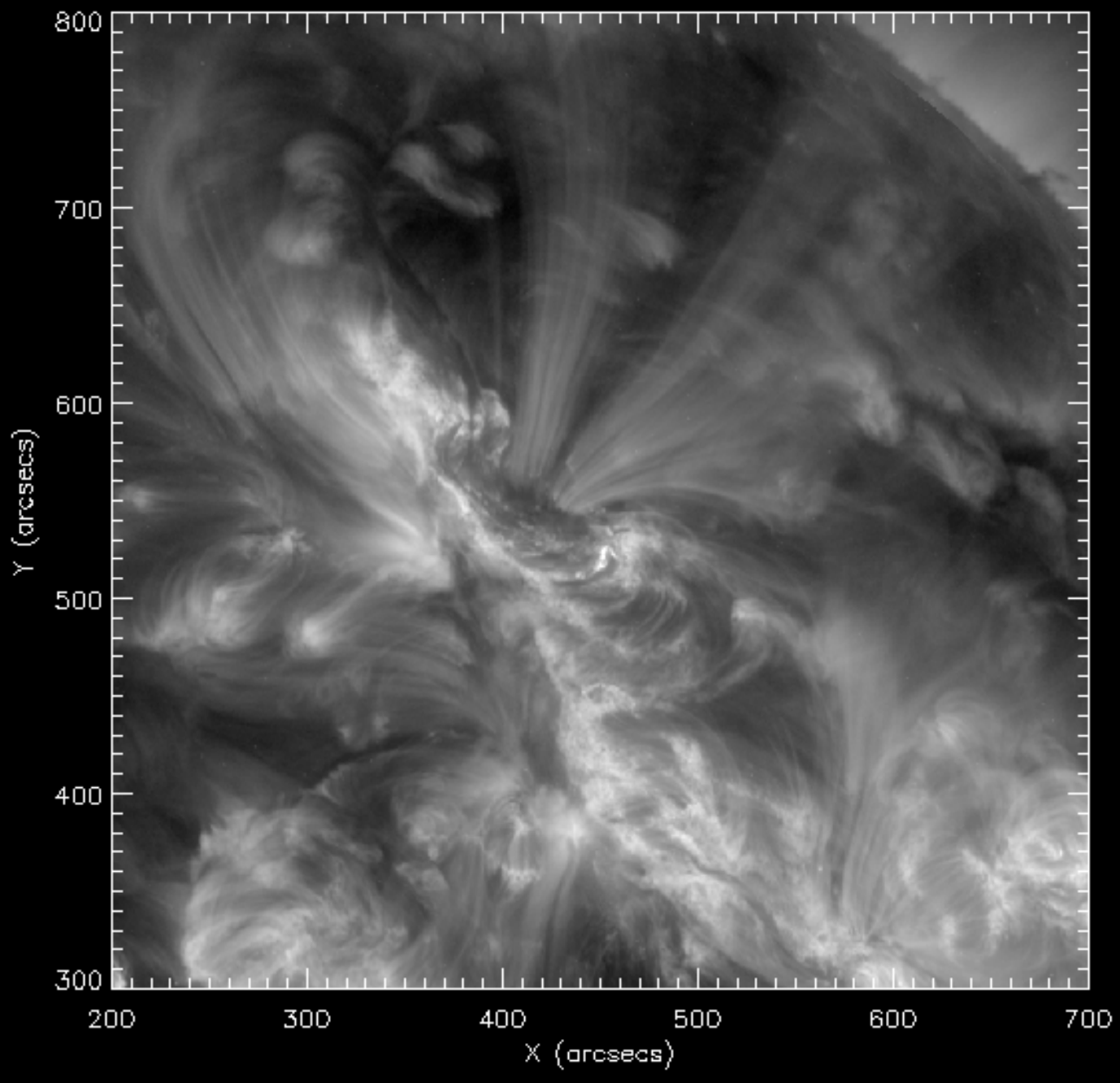

A. Sterling, Dec 2014

L5, Boulder 

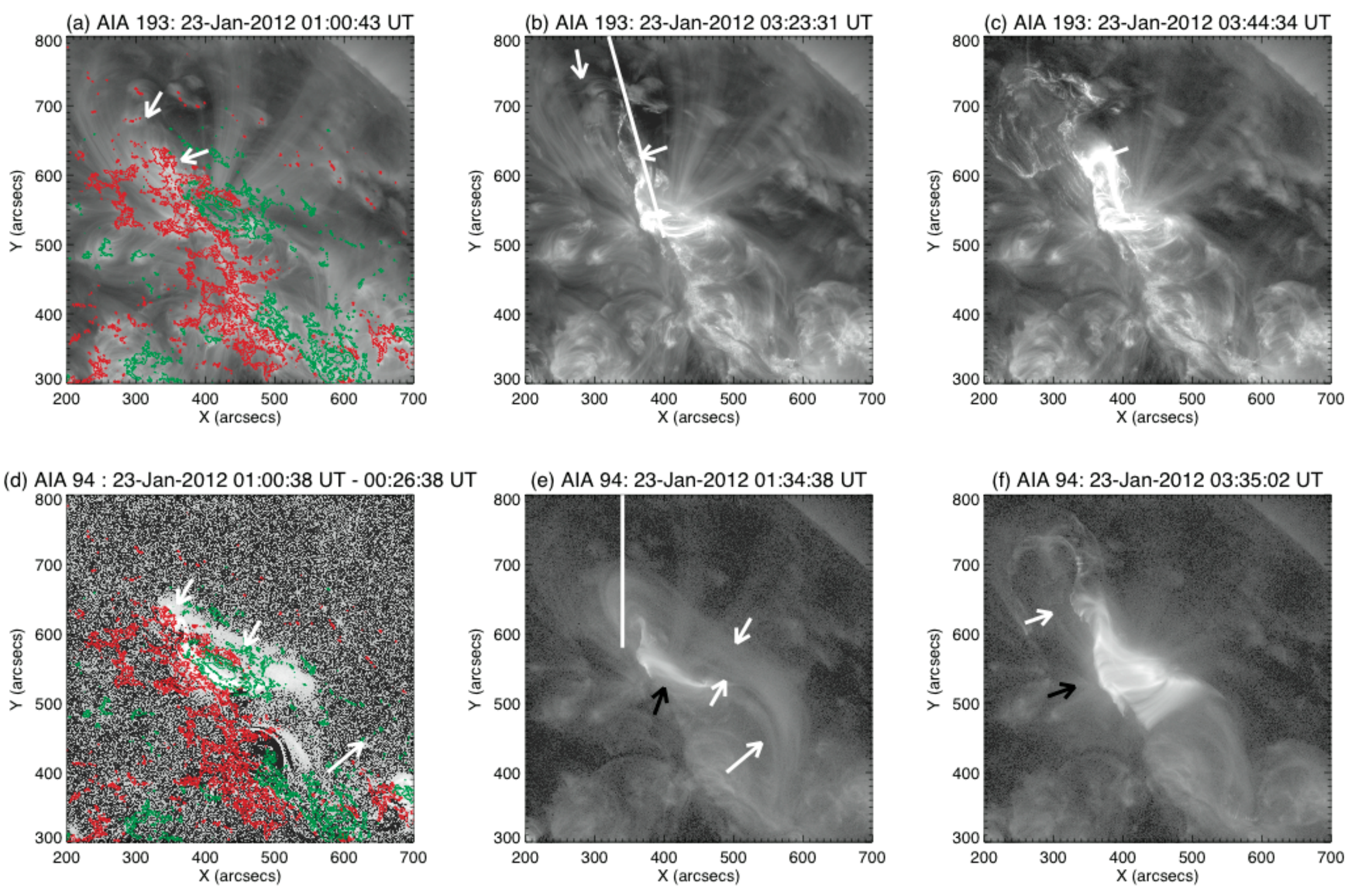

A. Sterling, Dec 2014
L5, Boulder 


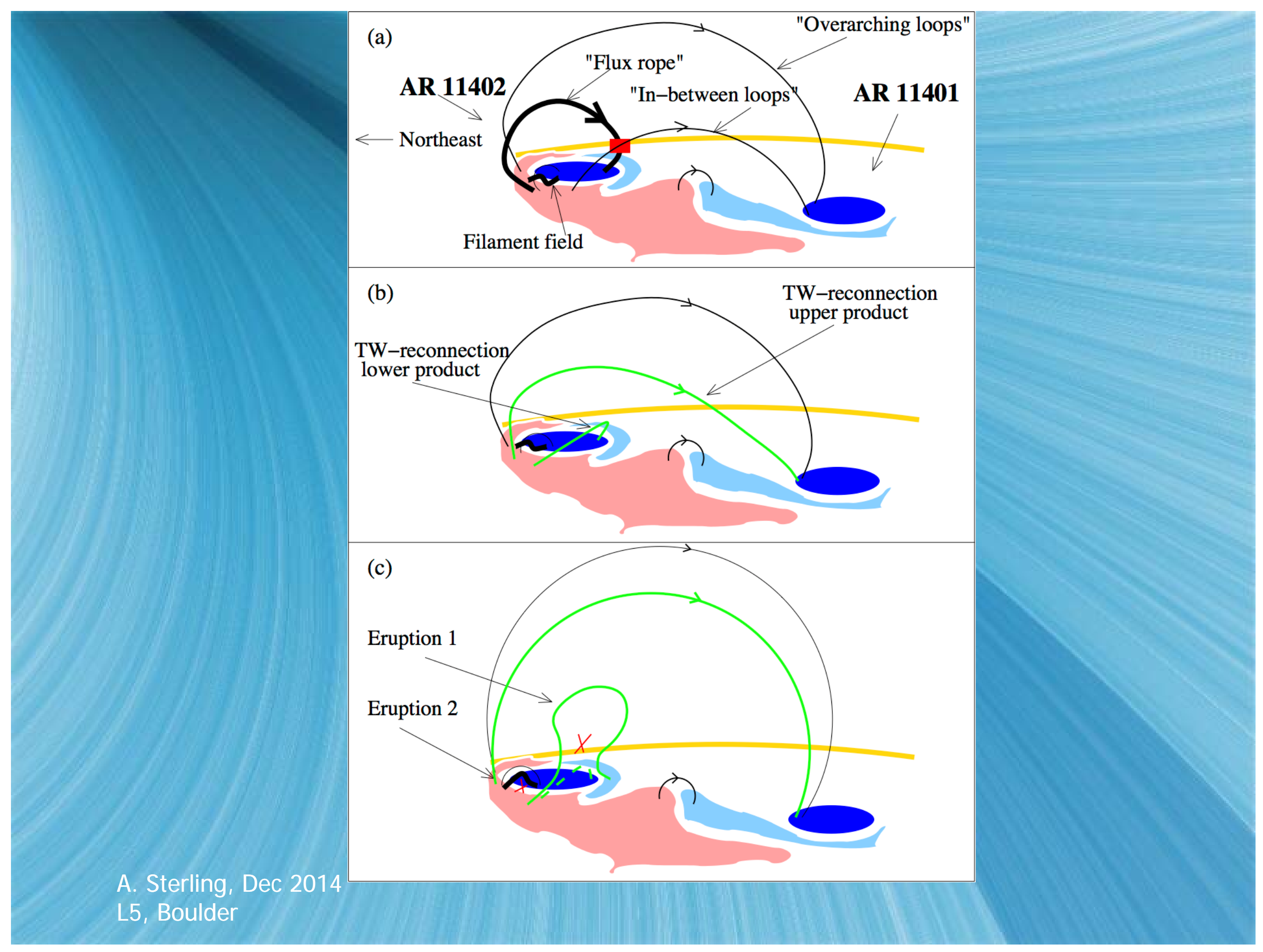




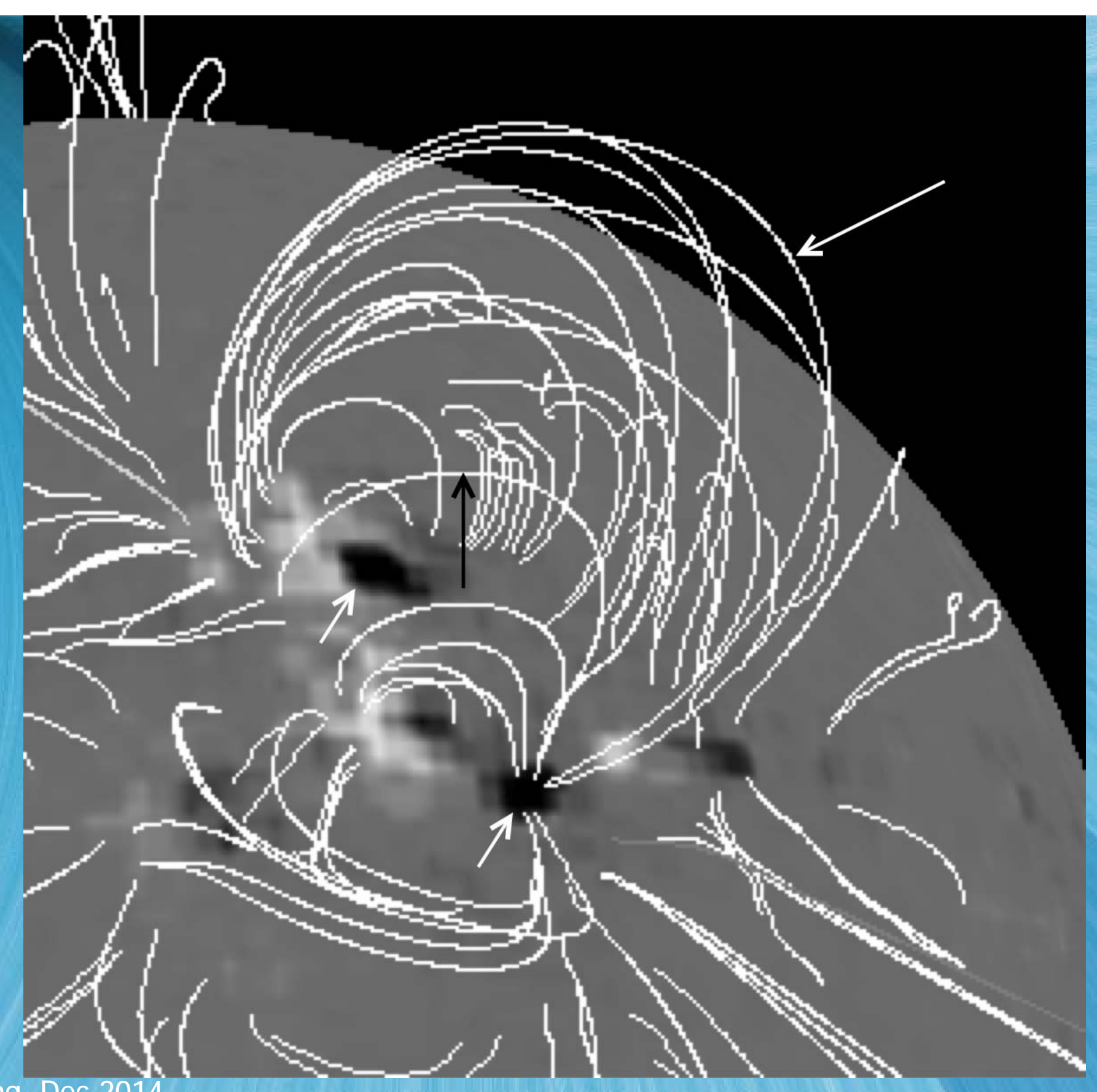

A. Sterling, Dec 2014

L5, Boulder 


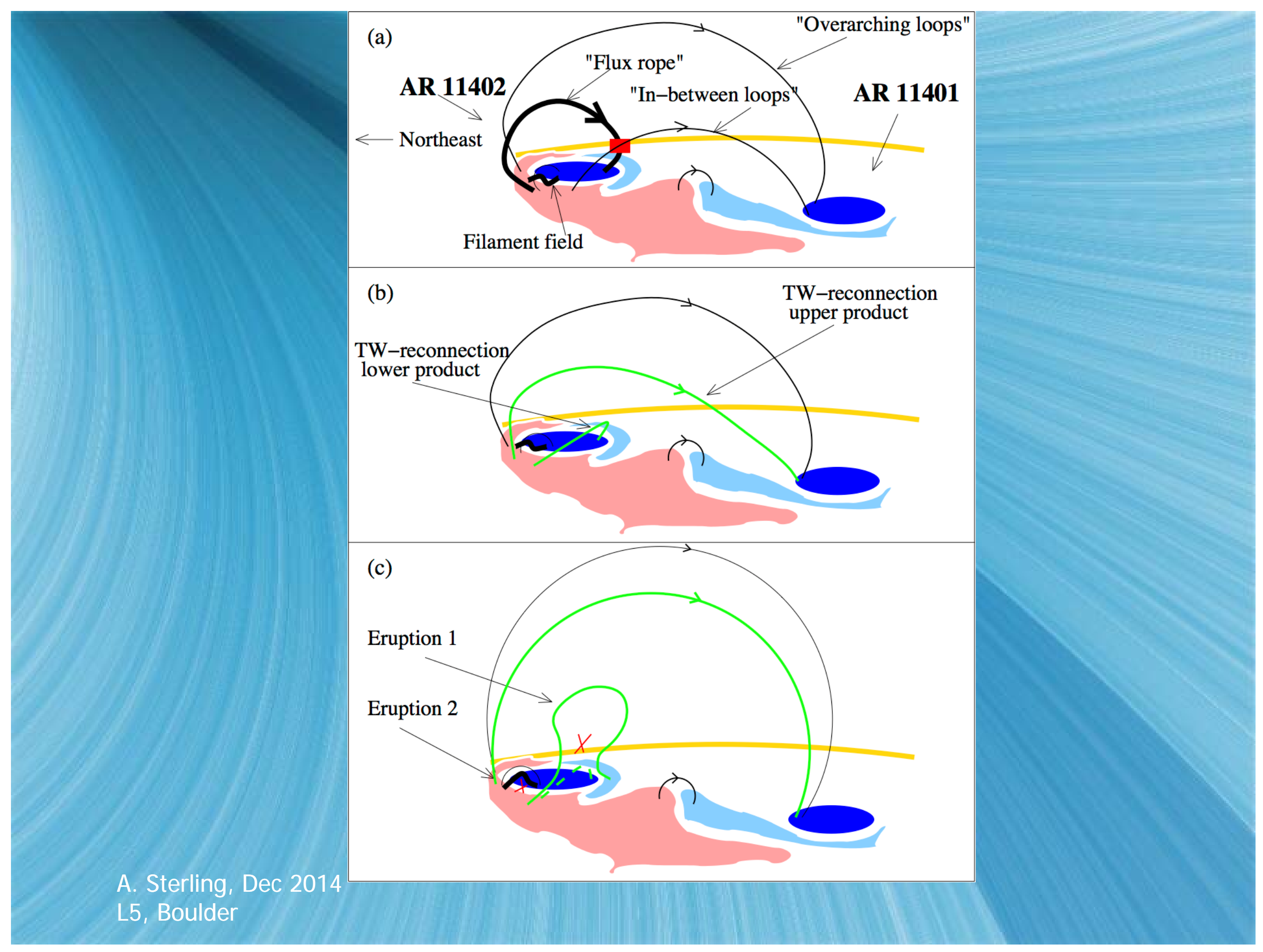




\section{Eruption 2 Via "Lid Removal"}

- Cheng et al. (2013) describe how Eruption 1 removes field above the Eruption 2 flux rope.

They show B gradient with height is steep enough for Eruption 2 flux rope to be subject to torus instability, allowing its eruption. (Eruption due to ideal MHD instability.)

We call their explanation for Eruption 2 (including filament) "lid removal."

Fundamentally different from eruption-trigger mechanisms we have examined (e.g., tether cutting, breakout...).

Similar however to other observations/descriptions (e.g., Schrijver \& Title 2011, Török et al. 2011).
A. Sterling, Dec 2014
L5, Boulder 


\section{Can Lid Removal Work with Eruption 1 Flare Arcade?}

- Standard flare model => Eruption 1 flare loops should form over filament arcade, perhaps preventing Eruption 2 ("confined eruption"). (S. Antiochos 2013, private comm.)

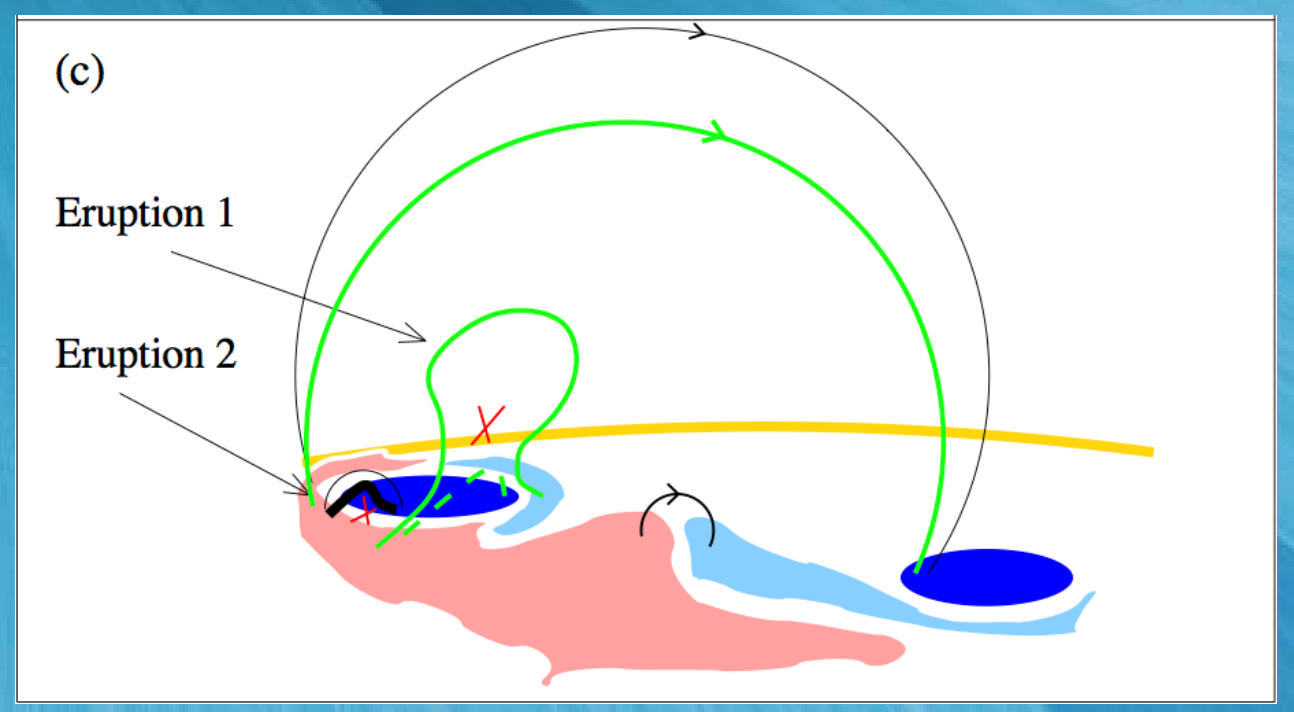

Look to see what happens.

Need "hot" AIA to see Eruption 1; need "cool" AI A to see filament. Use mixture; $70 \%$ hot (=131 Ang), 30\% cool (=193 Ang). 


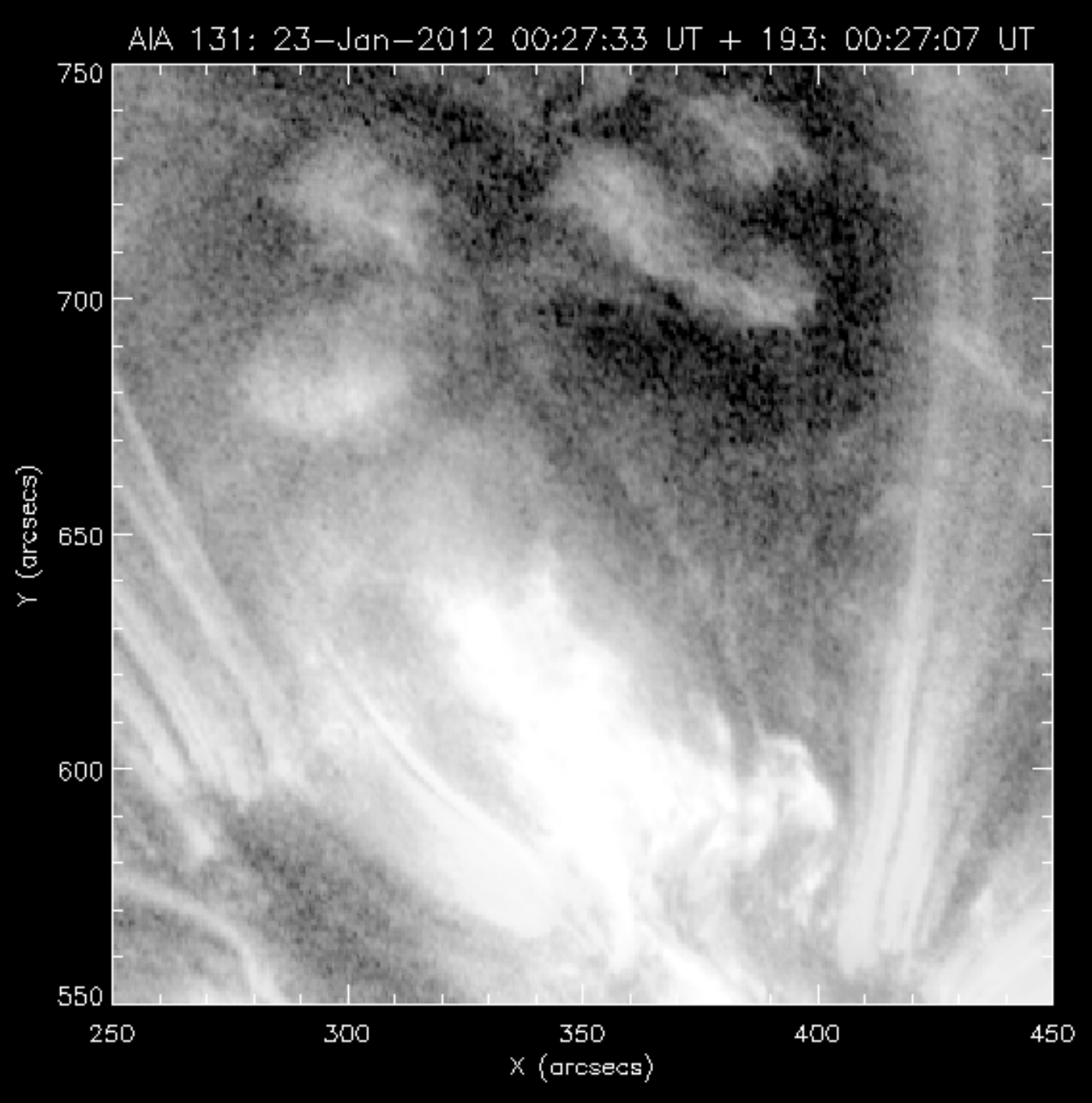

A. Sterling, Dec 2014

L5, Boulder 


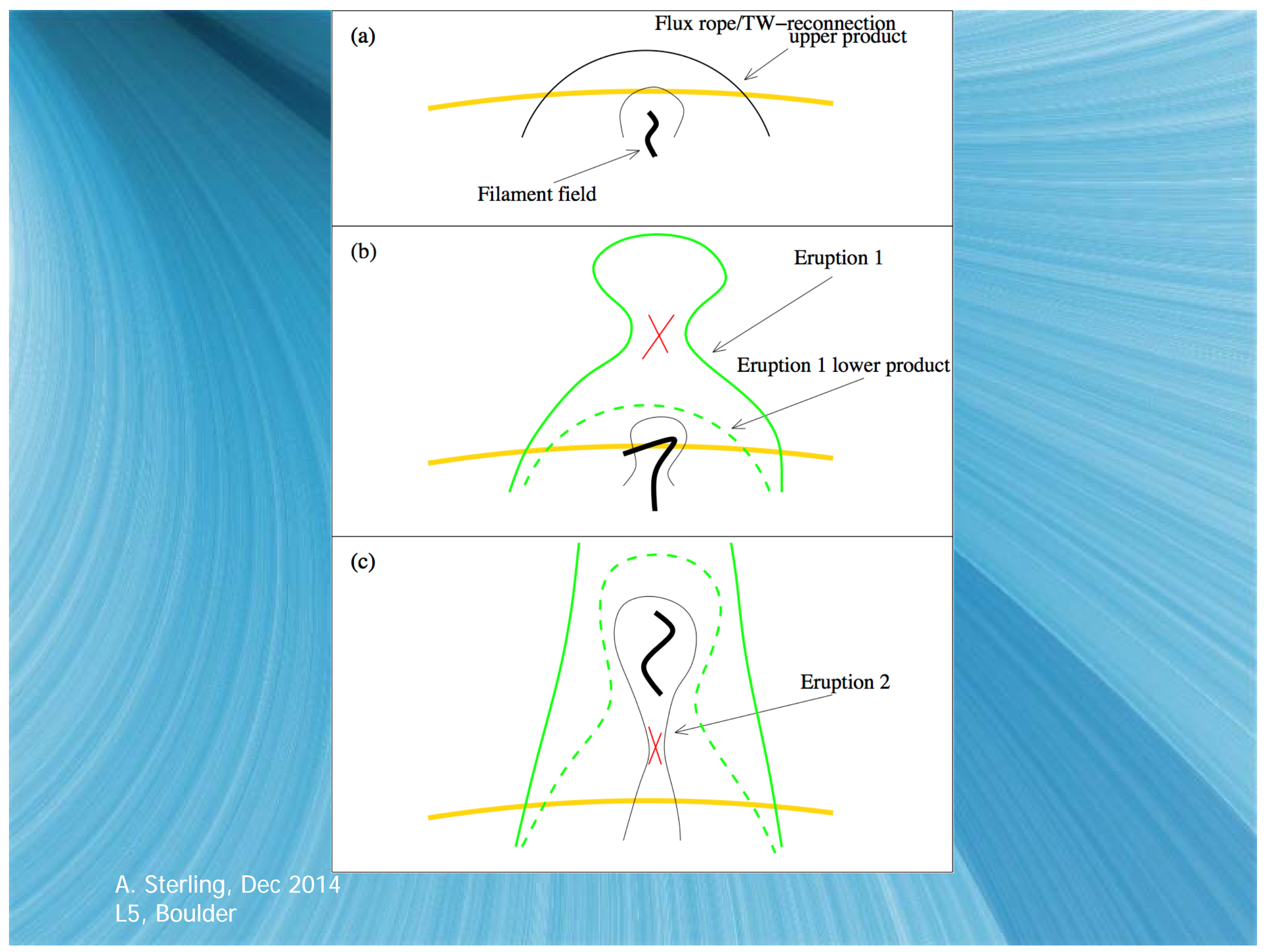




\section{Summary (2012 J an 23 event)}

- Two eruptions, with first only seen in AIA hot channels.

- Eruption 1 field reconnects with neighboring region, ("tether-weakening reconnection," Moore et al. 1992).

Eruption 1 removes field above filament arcade, leading to destabilization and onset of eruption 2; Lid Removal.

Eruption 2 blows out Eruption 1 flare loops.

Regarding SEPs: Double CMEs likely critical (e.g., Kahler 2001; Gopalswamy et al. 2002, 2003, 2004; Li et al. 2012). Effects on above points not yet known.

Sterling et al. (2014) provide more details. 


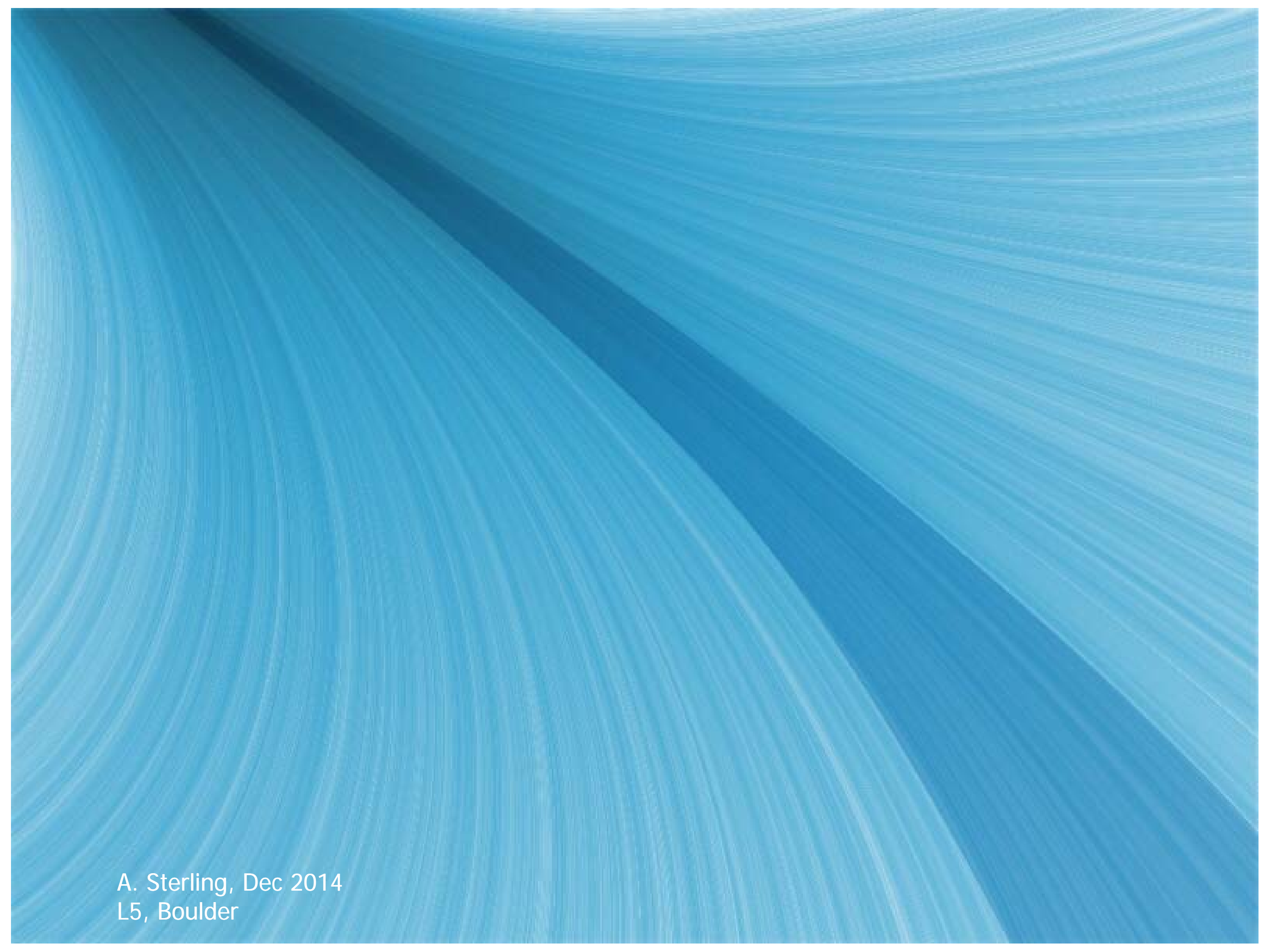




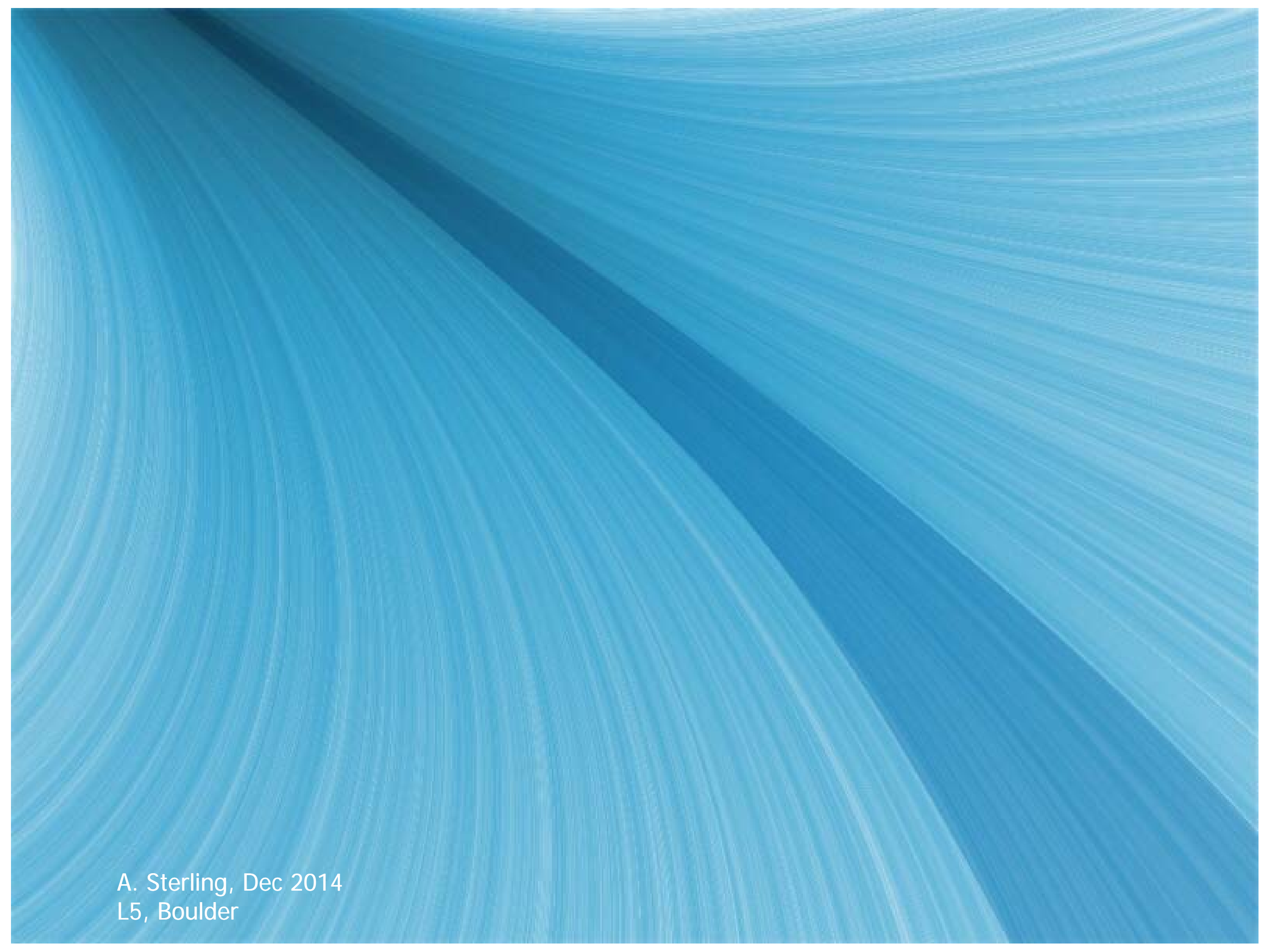




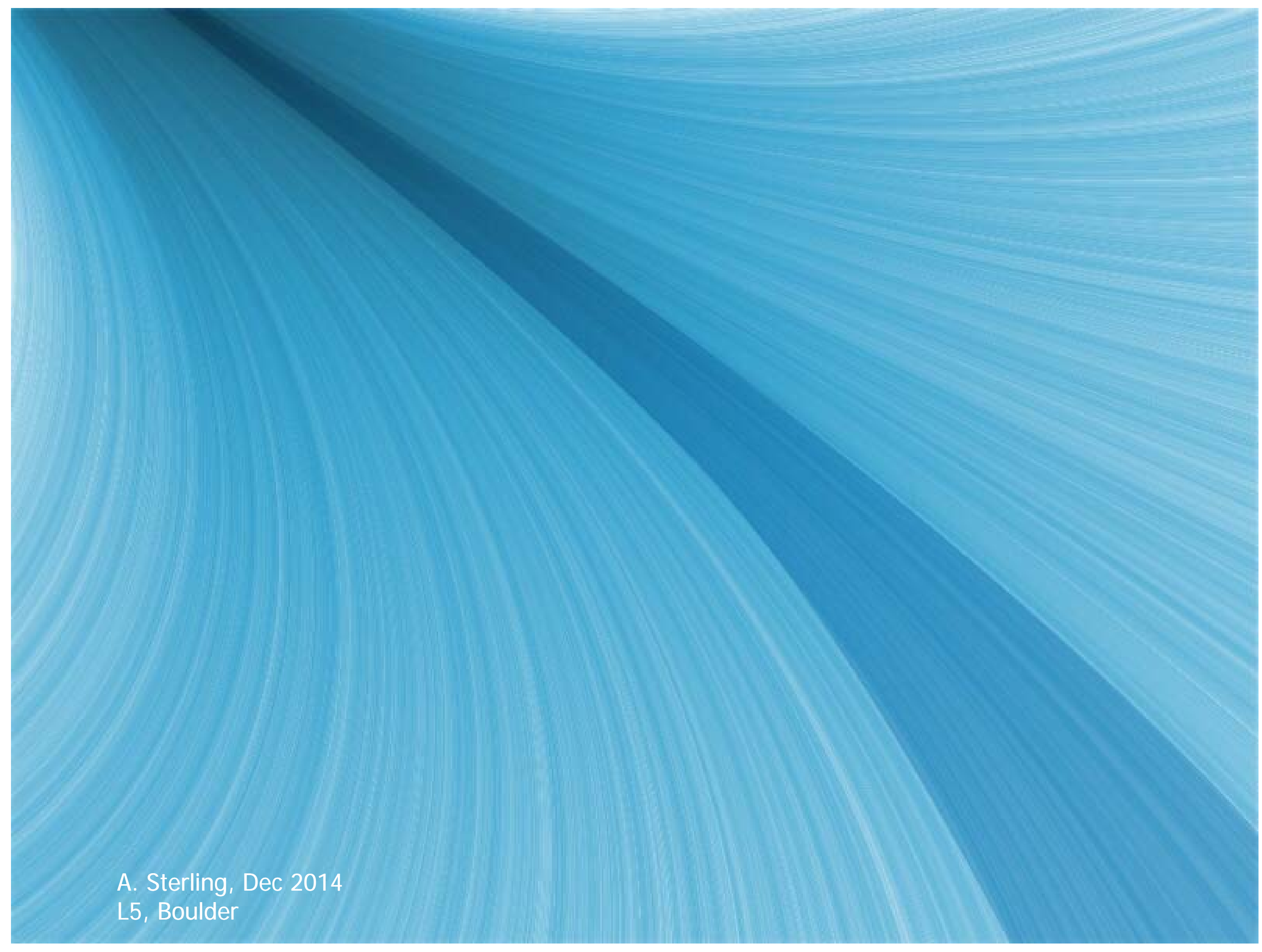



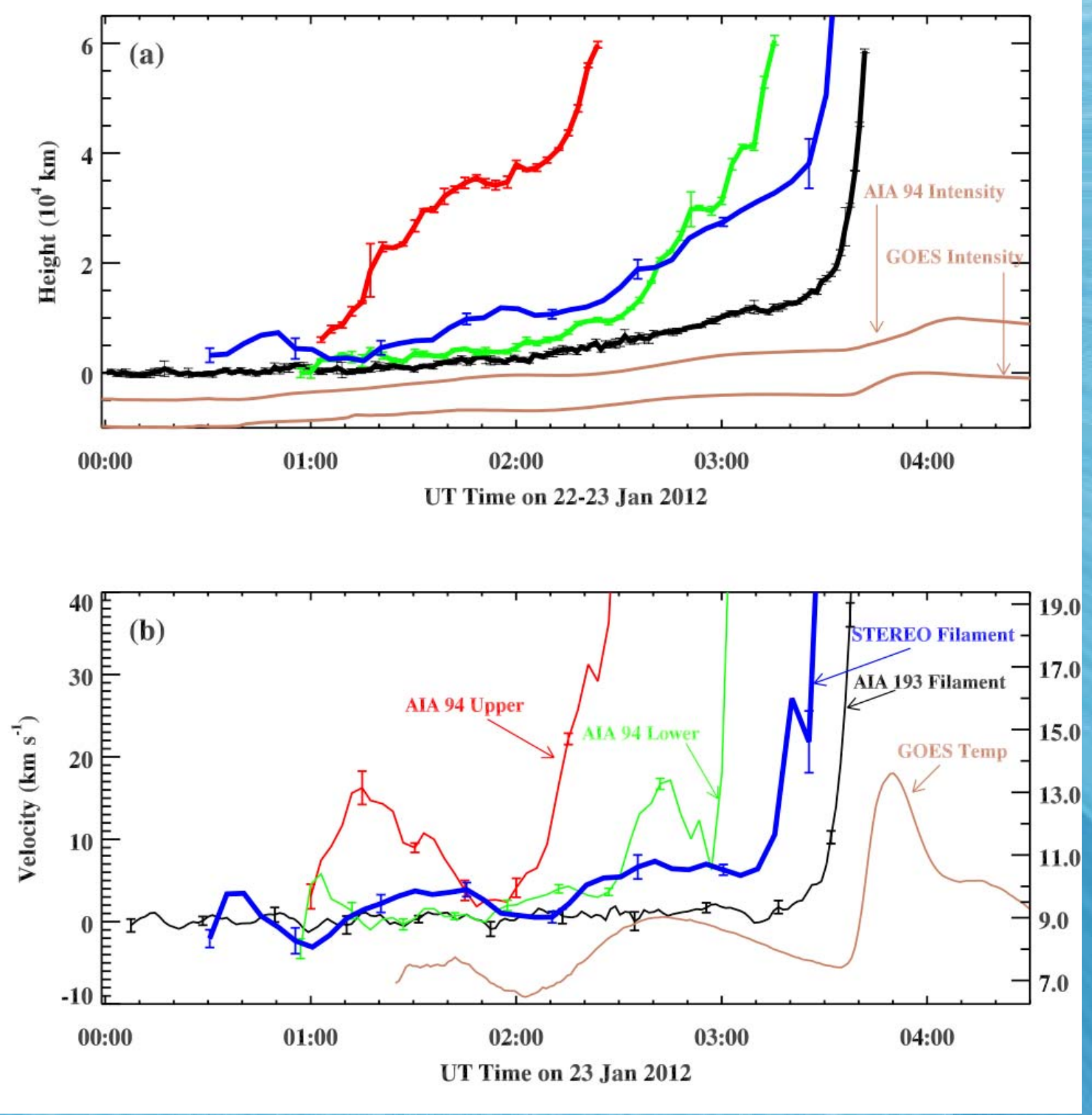

\section{A. Sterling, Dec 2014 L5, Boulder}




\section{What causes Eruption 1 onset?}


SDO/HMI HMI_FRONT2 6173 22-Jan-2012 15:15:25.600 UT

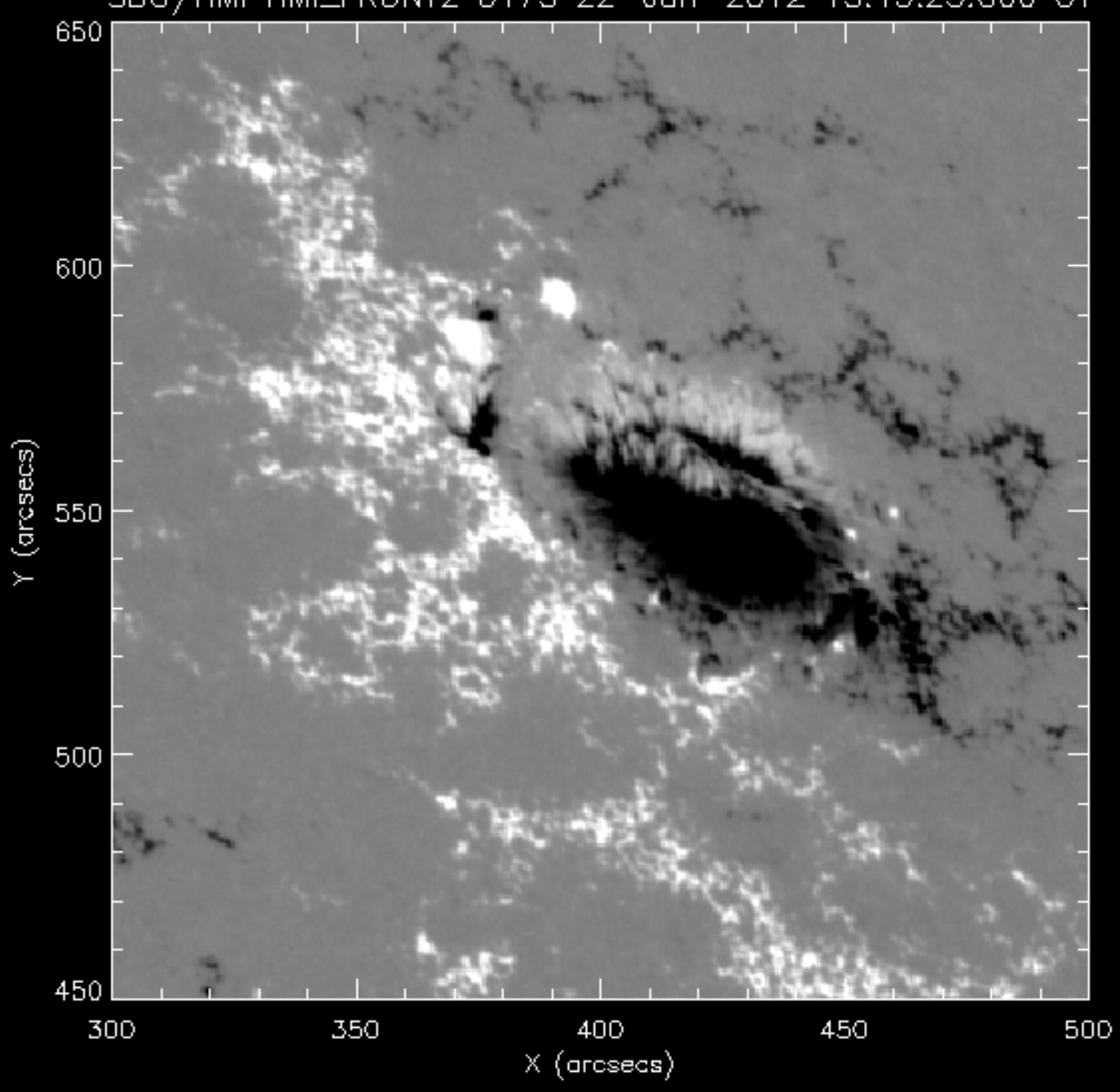

A. Sterling, Dec 2014 L5, Boulder 

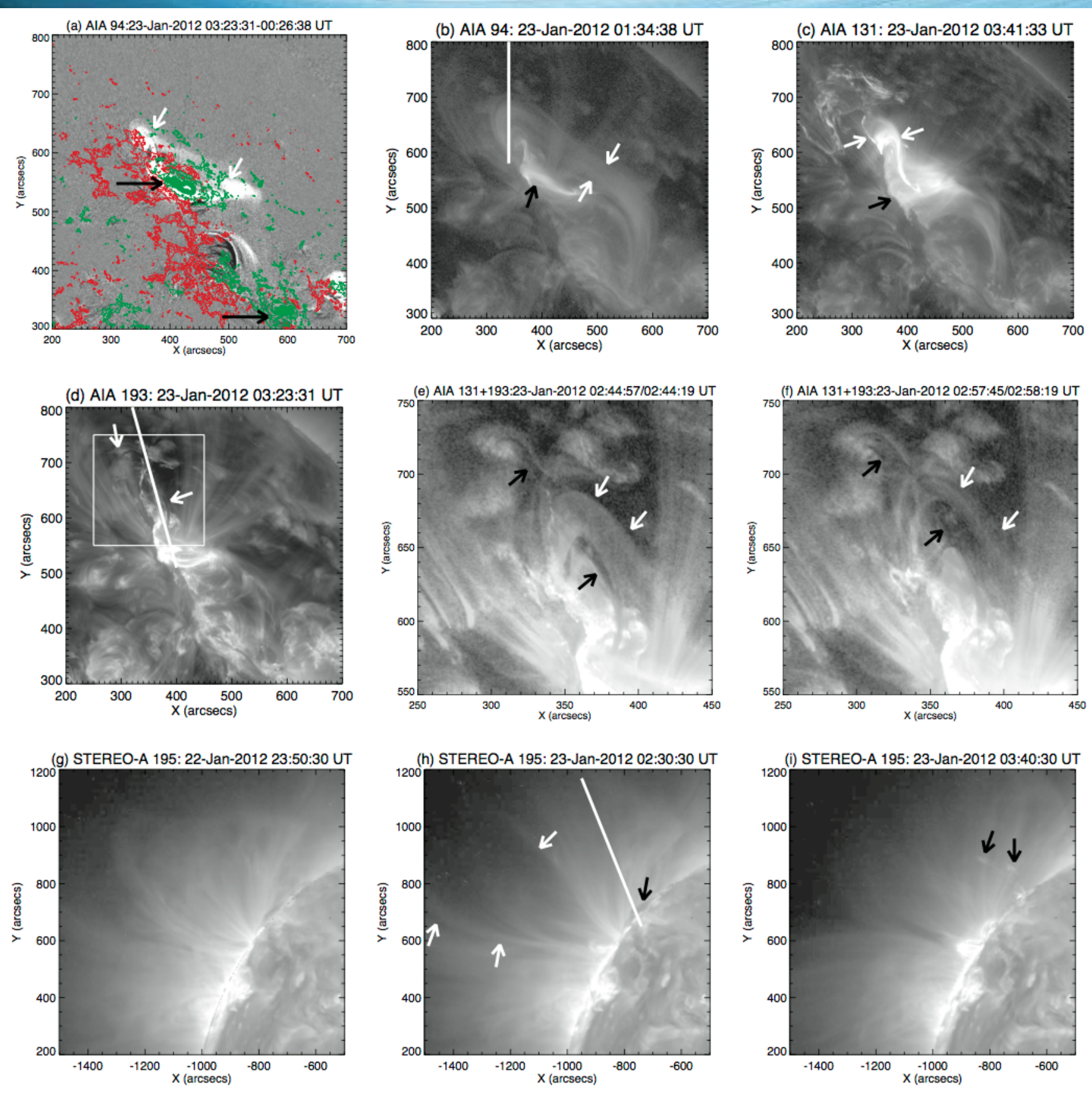

A. 5, Boulder 
SDO AlA_1 131 23-Jan-2012 00:26:09.620 UT

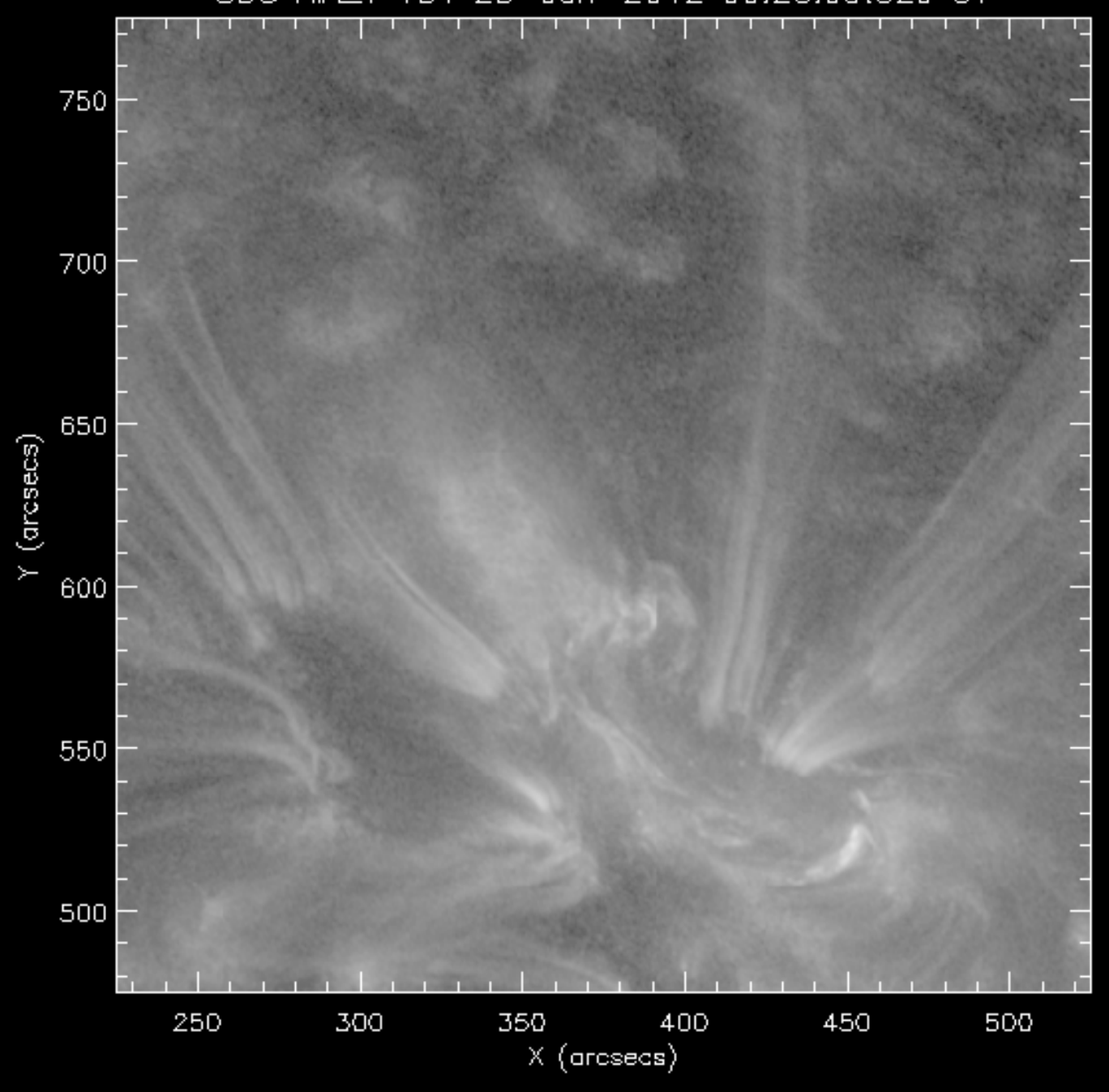

A. Sterling, Dec 2014

L5, Boulder 


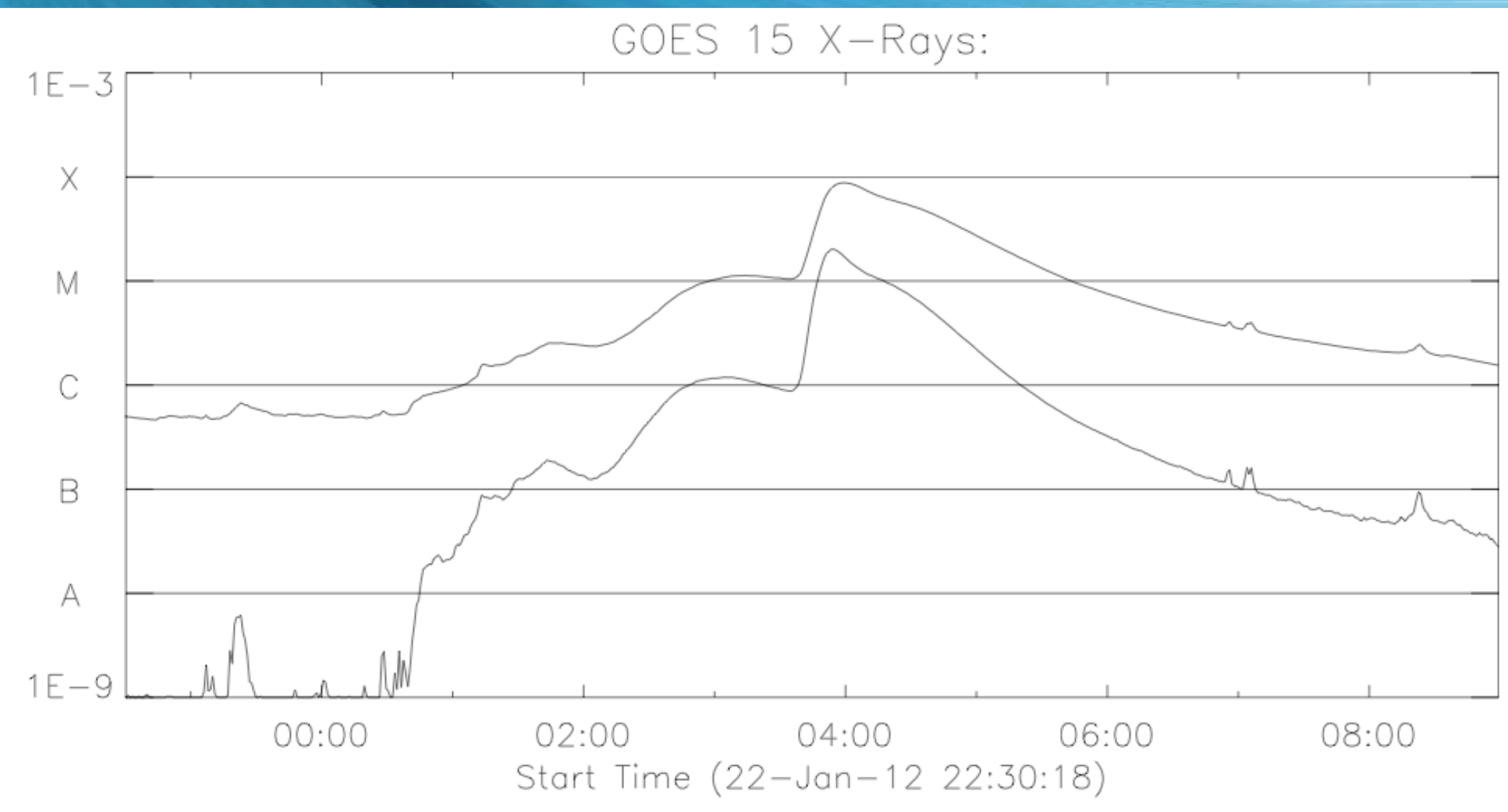

A. Sterling, Dec 2014

L5, Boulder 


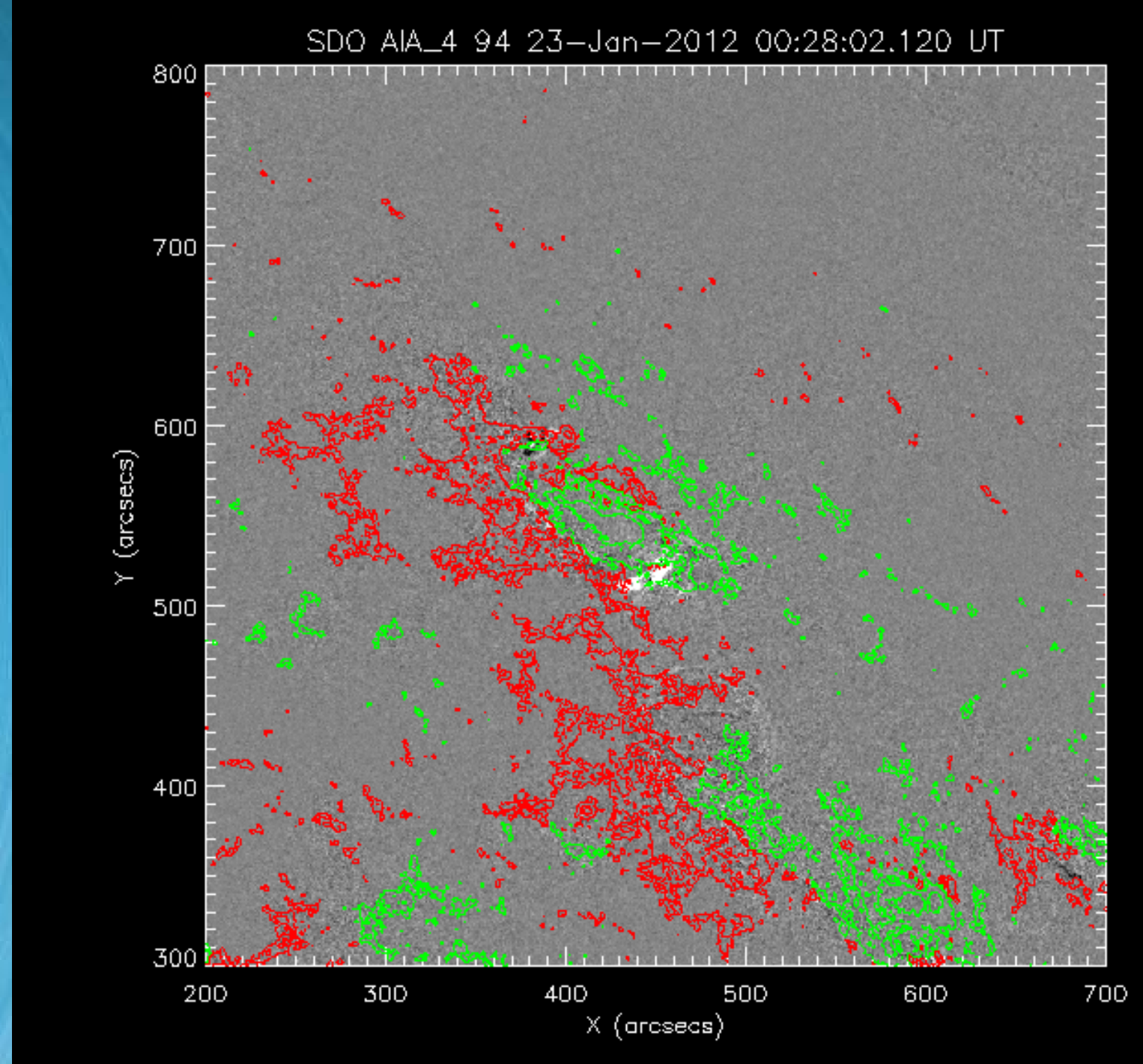

A. Sterling, Dec 2014

L5, Boulder 
STEREO_A SECCHI EUVI 195 22-Jan-2012 21:30:30.008 UT

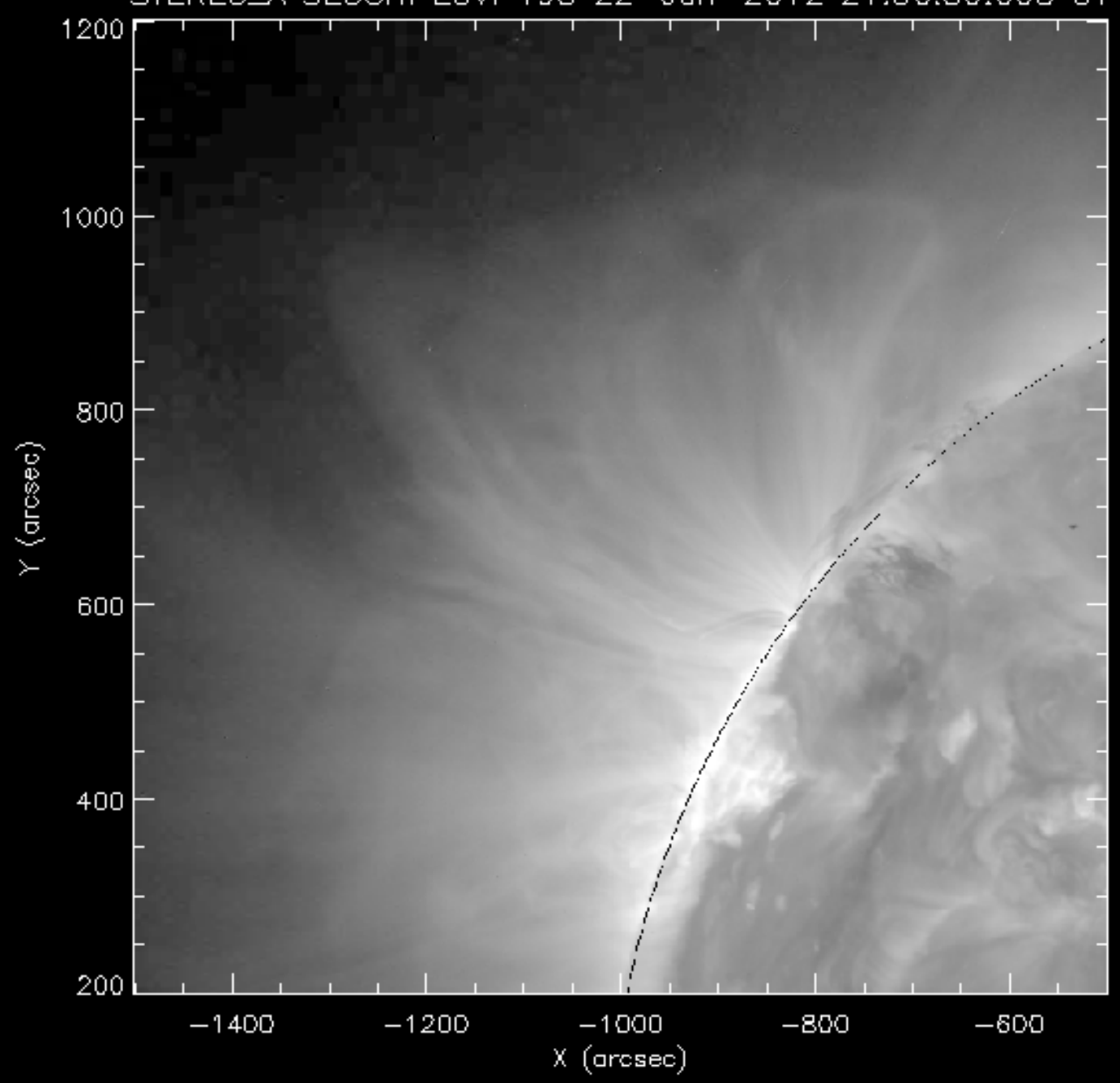

A. Sterling, Dec 2014

L5, Boulder 
(a) STEREO-A 195: 22-Jan-2012 23:50:30 UT

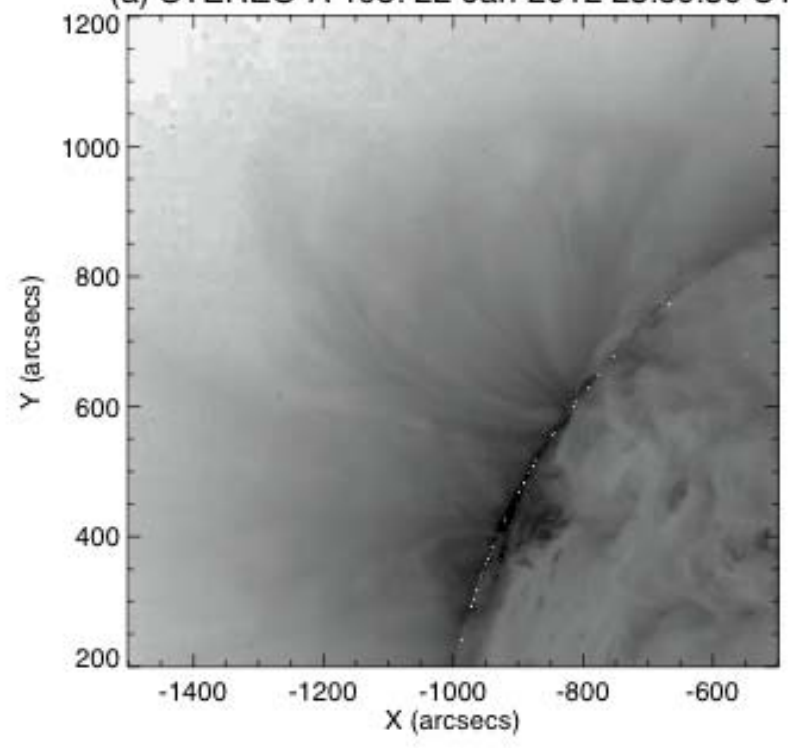

(b) STEREO-A 195: 23-Jan-2012 02:30:30 UT

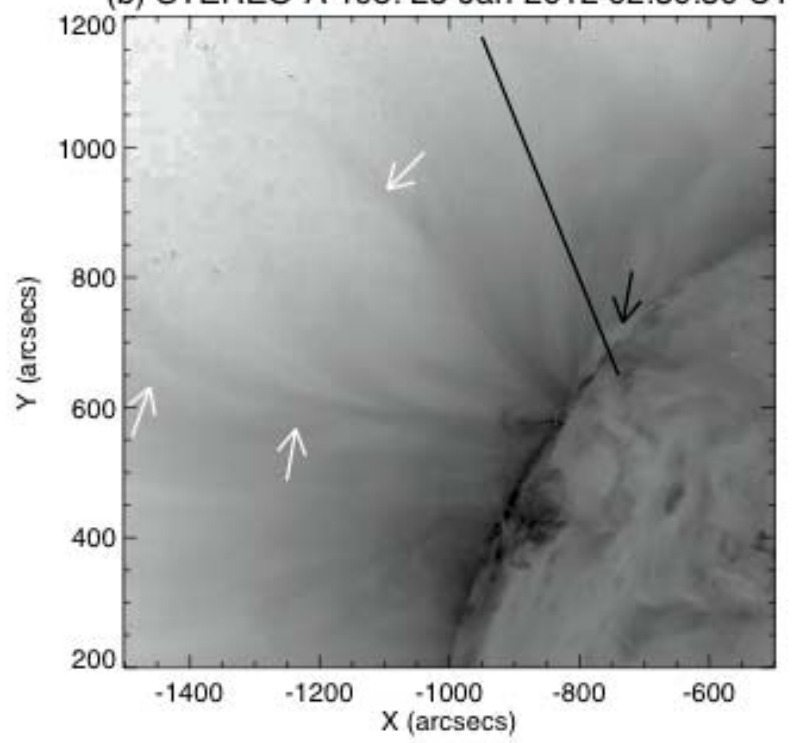

(c) STEREO-A 195: 23-Jan-2012 03:40:30 UT

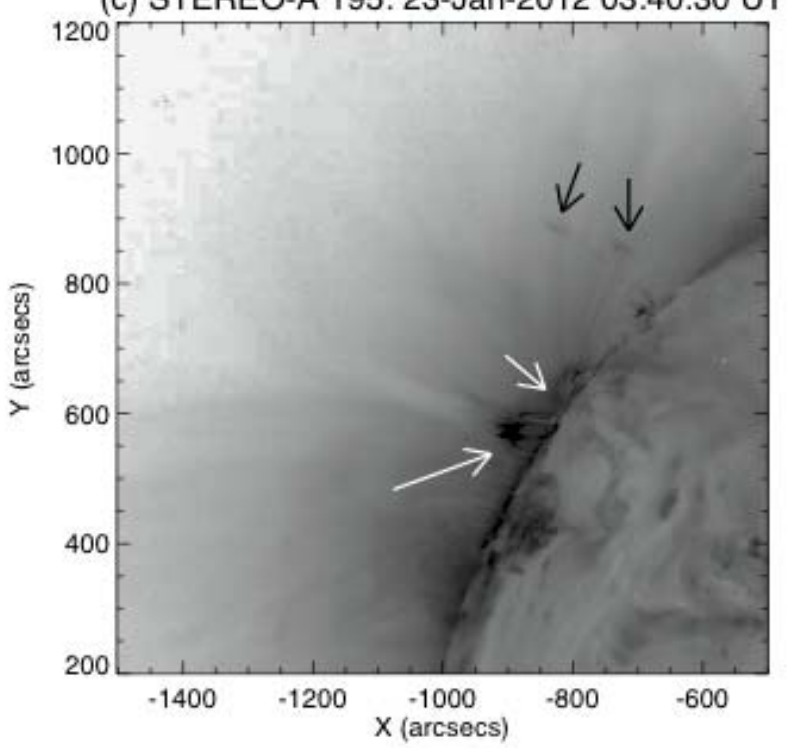

A. Sterling, Dec 2014

L5, Boulder 
- There are two eruptions ("eruption 1" and "eruption 2"), the latter including the filament. (Both components visible in LASCO CME images.)

- Eruption 1 nearly invisible in cooler on-disk EUV images, but appears as field opening in limb view (STEREO A).

- An even earlier brightening is due to "merging reconnection" (and eruption 1 flare).

- Filament eruption (eruption 2) is due to removal of overlaying flux by eruption 1 .

- This "Lid Removal" mechanism is a candidate for an ideal, non-resistive trigger. NB., it differs from the breakout mechanism.
A. Sterling, Dec 2014
L5, Boulder 


\section{Conclusions (2012 J an 23 event)}

- Something causes eruption 1; could be flux cancelation from MMFs.

- Eruption 1 field merges with neighboring region, with hotplasma signature (" merge reconnection").

- Eruption 1 removes field above filament arcade.

- This leads to destabilization and onset of eruption 2, creating the strongest and hottest GOES flare via Lid Removal.

Candidate for ideal onset mechanism.

Similar processes: E.g. Schrijver \& Title (2011); also Török et al. (2012). 